\title{
Roughening transitions of driven surface growth
}

\author{
Angel Sánchez \\ Theoretical Division and Center for Nonlinear Studies, Los Alamos National Laboratory, Los Alamos, New Mexico 87545 \\ and Departamento de Matemáticas, Escuela Politécnica Superior, Universidad Carlos III, \\ E-28911 Leganés, Madrid, Spain \\ David Cai, Niels Grønbech-Jensen, and A. R. Bishop \\ Theoretical Division and Center for Nonlinear Studies, Los Alamos National Laboratory, Los Alamos, New Mexico 87545 \\ Z. J. Wang \\ Theoretical Division and Center for Nonlinear Studies, Los Alamos National Laboratory, Los Alamos, New Mexico 87545 \\ and The James Franck Institute, 5640 Ellis Avenue, Chicago, Illinois 60637
}

(Received 17 January 1995)

\begin{abstract}
A model of surface growth given by a two-dimensional discrete, driven, damped sine-Gordon equation is studied using Langevin dynamics. Our large-scale simulations show that the equilibrium Kosterlitz-Thouless roughening transition splits into two crossovers (or transitions) under the external force of, e.g., vapor-surface chemical potential difference. Three different regimes are characterized in terms of roughness, growth rate, and height-height correlations - the onset of a rough phase is accompanied by the suppresion of oscillatory growth. Our results are interpreted consistently within a renormalization group framework. We discuss the generality of our conclusions and propose specific comparisons with experiments.
\end{abstract}

\section{INTRODUCTION}

Roughening transitions play significant roles in threedimensional (3D) crystal growth. Therefore, their study is very important both from fundamental and applied viewpoints. ${ }^{1}$ Equilibrium roughening transitions are by now well understood ${ }^{2}$ and most of them belong to the Kosterlitz-Thouless (KT) class. $^{3}$ Much less is known about the nature of the transition in the presence of external driving (corresponding, e.g., to the chemical potential difference between surface and vapor or to liquid undercooling when growing from melts). Kinetic roughening was first studied in Refs. 4-6 in the framework of the renormalization group (RG). A more careful microscopic analysis ${ }^{7}$ led to the conclusion that the transition was smeared by the driving, and experiments on hcp helium-4 (Ref. 8) were interpreted as supporting this conclusion. Recent analyses yielded basically the same conclusion $^{9}$ in spite of some disagreement on the details of the RG procedure. ${ }^{10}$ Our aim here is to gain insight into this problem by Langevin Dynamics (LD) simulations on large two-dimensional (2D) lattices.

With the above motivation, we consider the following $2 \mathrm{D}$ discrete sine-Gordon potential introduced in ${ }^{11}$

$$
V=\frac{1}{2} \sum_{\langle i, j\rangle}\left(\phi_{i}-\phi_{j}\right)^{2}-\sum_{i} \cos \phi_{i}
$$

with $\phi_{i}$ continuous variables on a square lattice. The first term describes the interaction energy between a column at site $i$ and its nearest neighbors, representing surface tension, and the second term favors $\phi_{i}$ to be $2 \pi n$ (so we can identify $\phi=2 \pi h, h$ being the height) and is di- rectly related to periodic pinning potential effects of the lattice. The equilibrium roughening temperature was determined in Refs. 11 and 12, in very good agreement with RG predictions. Furthermore, recent experiments ${ }^{13}$ on growth of GaAs by metalorganic vapor phase epitaxy exhibit morphologies very similar to those reported in Ref. 11. These data suggest that this model captures much of the essential physics of surface growth phenomena.

The remainder of the paper is organized as follows. In Sec. II, we summarize our simulation procedure, namely, Langevin molecular dynamics. Subsequently, we present our results in detail, discussing the dependence of growth rate, roughness, and height-height correlation function on temperature. By this means, we are able to characterize the behavior of our model in terms of three different regimes, thus providing a quite comprehensive picture of its dynamics. In Sec. III, we discuss our results in the framework of RG ideas, and consistently interpret our findings in terms of competition between renormalized driving strength and renormalized periodic potential. All of these considerations lead us to the conclusion that the equilibrium $\mathrm{KT}$ transition splits in two rapid crossovers, which we summarize in Sec. IV. We close this section and the paper by comparing our results on our surface growth model to similar phenomena in related models, and by proposing how our predictions can be experimentally verified.

\section{LANGEVIN MOLECULAR DYNAMICS RESULTS}

We study model (1) by LD, i.e., by numerically integrating the coupled Langevin equations of motion for $\phi_{i}$, 


$$
\ddot{\phi}_{i}-\alpha \dot{\phi}_{i}=\sum_{\langle i, j\rangle}\left(\phi_{j}-\phi_{i}\right)+\sin \phi_{i}+I+\xi_{i}(t),
$$

where dot means time derivative, $I$ represents the driving, and $\xi_{i}(t)$ is a Gaussian white noise of zero mean and variance $\left\langle\xi_{i}(t) \xi_{j}\left(t^{\prime}\right)\right\rangle=2 \alpha T \delta_{i j} \delta\left(t-t^{\prime}\right)$, in units such that $k_{B}=1$. A dissipation coefficient $\alpha$ has been introduced with the requirement that we are in the overdamped regime. Our numerical procedure is a fast ${ }^{14}$ implementa- $^{2}$ tion of a stochastic Runge-Kutta method, ${ }^{15}$ which allows the study of very large lattices on workstations, without resorting to a massively parallel computer. ${ }^{11}$ These major computational advances make LD an attractive method for nonequilibrium statistical mechanics problems, such as surface growth and many other condensed matter issues. ${ }^{16}$ In particular, it allows us to realistically explore mesoscopic dynamics, as well as simple thermodynamic quantities. We integrated Eqs. (2) for long times, up to $10^{4}$ time units, for a number of values of temperature, driving, and dissipation. $L \times L$ lattices up to $L=1024$ sites with periodic boundary conditions were used, most of the simulations being performed on $L=256$ systems after it was verified that our main results did not depend strongly on $L$.

Figure 1 collects the results of our simulations for the growth rate. The instantaneous growth rate, $v(t)$, is defined as the lattice average of $\dot{\phi},\langle\dot{\phi}\rangle_{l}$, and the growth rate, $v$, is the average of $v(t)$ over the duration of the run excluding transients. Three regimes are clearly distinguishable: Regime I (low temperature), where the surface does not grow on the timescale of our simulations; regime II (middle temperature), where growth is nonlinear with respect to the driving (i.e., $v<\alpha I$ ); and regime III (high temperature), where $v \sim \alpha I$. Results for very different (overdamped) dissipation values are qualitatively the same. The crossover between regimes II and III can be estimated (data for $I=0.01$ are not very reliable be-

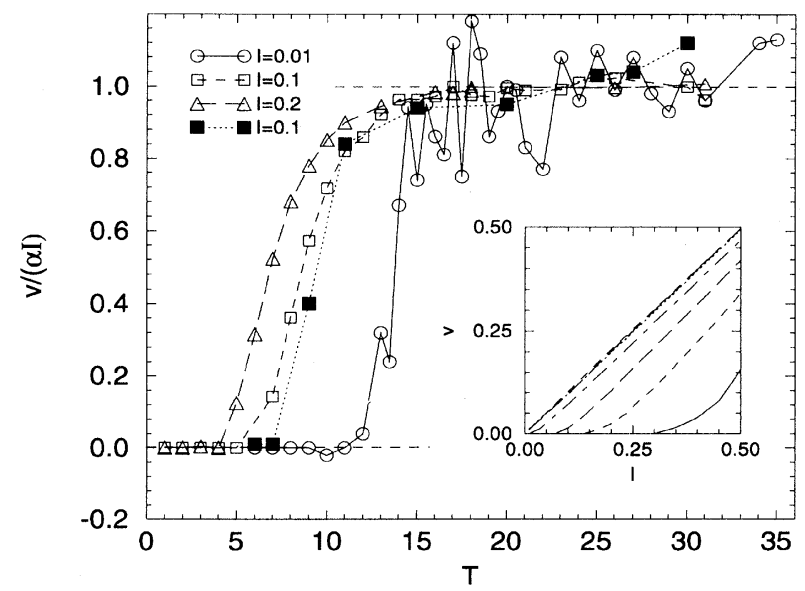

FIG. 1. Scaled growth rate $v /(\alpha I)$ vs temperature. Driving values are as indicated in the figure. Dissipation is $\alpha=1$ (empty symbols) or $\alpha=10$ (full squares). The inset is $v$ vs $I$; lines are isotherms for $T=3,5,7,10,18,26,31$ from the bottom to the top (the last two on top of each other). cause fluctuations of $v$ are of the order of magnitude of $v$ itself) to occur around $T=8 \pi$. In our units, this is the equilibrium roughening temperature. ${ }^{11} \mathrm{On}$ the other hand, the transition from regime I to regime II is quite sharp for all values of $I$.

The inset in Fig. 1, where isotherms are plotted, sheds light on the three regimes: Thus, the II $\rightarrow$ III crossover is revealed in this plot by the superposition of all isotherms above $T=8 \pi$, where the growth rate is linearly proportional to the driving for all values of $I$ [i.e., the free Ohmic response regime, $v=\alpha I$ (Ref. 16)]. Below this temperature, isotherms tend asymptotically to this $T=8 \pi$ one with increasing driving. Finally, regime II is seen to have two subregimes separated by a smoother crossover (associated with the marked change in slope in the $v-T$ plot, see Fig. 1): For low temperatures, $v$ is not linear in $I$, while higher temperatures exhibit linear behavior (but not the free Ohmic response of regime III). Regime II is analogous to one-dimensional (1D) nucleation of solitons $;^{17}$ in terms of that physical picture, for temperatures just above the $\mathrm{I} \rightarrow \mathrm{II}$ transition growth is field induced, becoming thermally induced for higher temperatures. As for regime I, the fact that for the very long times of our simulations the surface does not grow agrees with the expected extremely small probability of thermally creating a critical nucleus in $2 \mathrm{D}$.

Figure 2 shows the signatures of the three regimes in the roughness, $w^{2} \equiv\left\langle\left(\phi_{i}^{2}-\left\langle\phi_{i}\right\rangle_{l}^{2}\right)\right\rangle_{l}$. Plotted values are those of $w^{2}$ at $t=700$, a long time after transients have ceased; longer runs give the same results. The most striking feature is the presence of a well-defined jump in the roughness dependence on temperature. The temperature at which this jump takes place decreases with increasing driving and coincides with that of the $\mathrm{I} \rightarrow \mathrm{II}$ crossover for all values of $I$. Remarkably, the temperature dependence of roughness in region $I$ is independent of the driving within an accuracy better than $1 \%$. After the jump, in region II, there are clearly visible oscillations in the roughness dependence on $I$. The origin of these oscillations can be understood by examining the

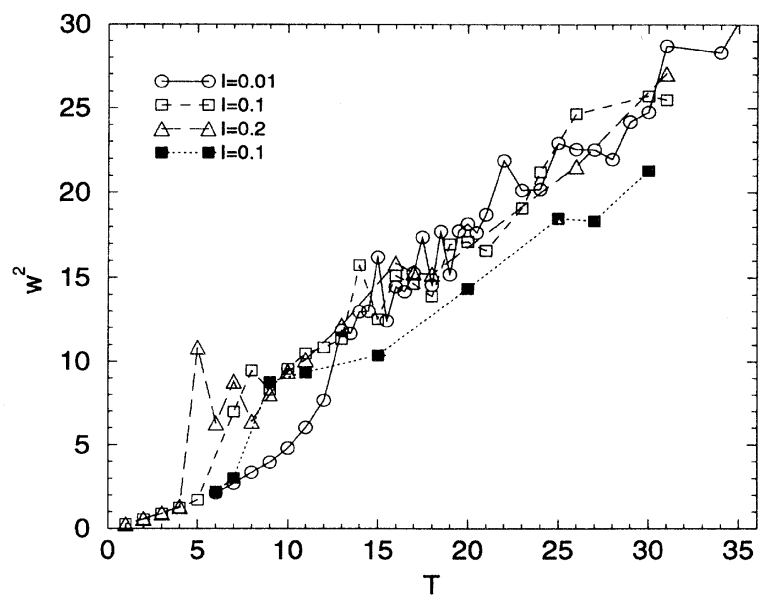

FIG. 2. Roughness $w^{2}$ vs temperature for the same drivings and dissipation as in Fig. 1. 
simulation for a case in region II shown in Fig. 3. Note that in this regime, height is approximately linearly proportional to time, see inset in Fig. 3; however, it can also be appreciated from the plot that the growth is not truly linear but has an oscillatory component. The same phenomenon is found everywhere in region II, with both the period and the amplitude of the oscillations decreasing with increasing temperature. It is very important to note that the evolution of roughness in this regime is not independent of that of the height, as demonstrated in Fig. 3: Maxima (minima) of the roughness always occur shortly after the surface crosses $(2 n+1) \pi(2 n \pi)$. This provides an insight to the growth mechanism operating in this region, which is confirmed by direct plots of the surface height profile. Namely, growth from $2 n \pi$ proceeds by developing fluctuations, which reach the immediately following potential minimum, thus giving rise to an increase of the roughness. Driving is responsible for these fluctuations appearing preferentially in the direction of the growth. When there are many parts of the surface at the next minimum, they help raise the rest of the surface to that height, leading to a decrease in the roughness. The analogy with $1 \mathrm{D}$ nucleation theory ${ }^{17}$ is evident again and, correspondingly, oscillatory growth becomes undistiguishable from thermal fluctuations when crossing over from field-induced to thermally induced growth.

Further information can be extracted from the height difference correlation function, $C(r) \equiv\left\langle\left[\phi\left(r+r^{\prime}\right)-\right.\right.$ $\left.\left.\phi\left(r^{\prime}\right)\right]^{2}\right\rangle$, which is power law above the roughening temperature and exponential, with finite correlation length, below the equilibrium transition. ${ }^{1,2}$ Figure 4 shows that in region I correlations are of finite range, saturating rapidly with distance. Moreover, the correlation function does not depend (after an initial transient) on the duration of the run, i.e., it does not evolve in time. On the other hand, in regions II and III correlations show powerlaw behavior for small $r$. Simulations for the highest temperatures, near the II $\rightarrow$ III transition, support the fact that the correlation is still evolving in time and the correlation length is developing towards its maximum, the

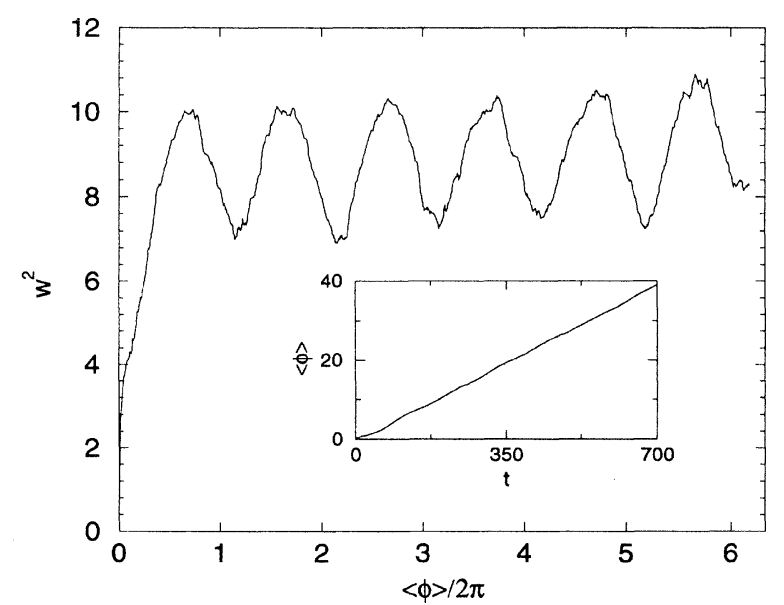

FIG. 3. Roughness vs surface height for $I=0.1, \alpha=1$, and $T=7$. The inset is height vs time for the same simulation.

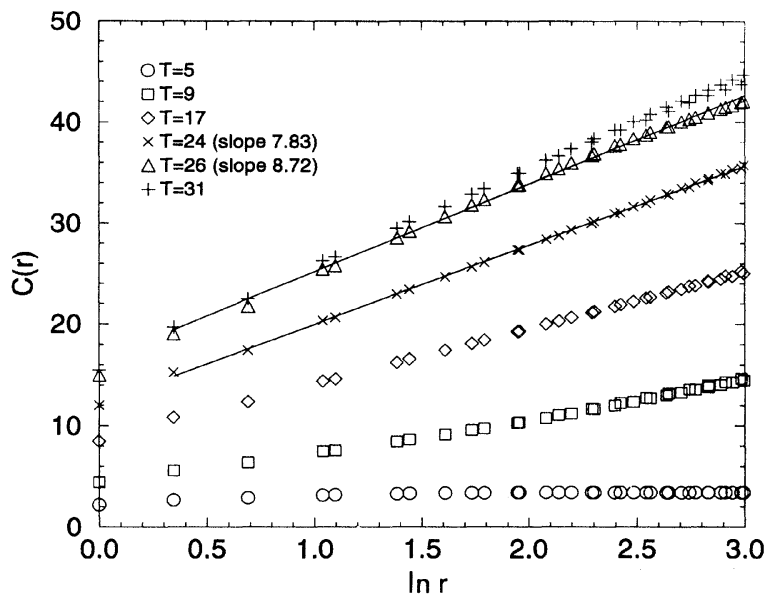

FIG. 4. Height difference correlation functions $C(r)$ for $I=0.1$ and $\alpha=1$, for different values of temperature.

system size. On the contrary, close to the $\mathrm{I} \rightarrow \mathrm{II}$ crossover, numerical results indicate that the correlation length is finite. Importantly, at the II $\rightarrow$ III crossover temperature, the slope of the correlation function passes through the universal value 8 (in our units) (Refs. 4 and 11) related to the equilibrium roughening transition. From our simulations, this value turns out to be almost independent of the temperature in a large range of driving forces (see Fig. 1), suggesting that the II $\rightarrow$ III crossover is insensitive to the driving.

\section{DISCUSSION}

We now attempt to understand the above numerical findings on the basis of previous theoretical work. ${ }^{4-10}$ We propose that the appearance of the three regimes arises from the competition of different terms in interaction (1) for dominance under renormalization. Thus, the $I \rightarrow I I$ transition corresponds to the (renormalized) driving strength being such that it allows the surface to overcome the (thermally softened) periodic potential. To use a simple analog, it is the inflection point where the tilt of a washboard allows a marble to begin running down. This interpretation is consistent with the fact that the crossover temperature decreases with increasing driving: Larger drivings are able to overcome larger potential barriers. In regime I, the periodic pinning potential is the dominant effect, and therefore, the results should be independent of the driving, as indeed is the case. Then we enter region II. The motion of the surface, in spite of being able to evolve from one well to the next and grow, is still not free, as the effective potential is not renormalized to zero. This is why growth in this region shows oscillations and the growth rate increases with increasing temperature. Furthermore, the "washboard picture" is directly connected with the oscillations in height and roughness reported above: The surface, although it never stops growing, stays longer in the (still present, but metastable) minima of the potential, and its 
motion is assisted by fluctuations; thus, the oscillatory behavior characteristic of field-induced growth. As temperature increases, the effective potential is smaller, and the influence of thermal fluctuations becomes more visible, leading to disappearance of the oscillations into thermally induced growth. Eventually, temperature reaches a value around $8 \pi$ at which the periodic potential renormalizes to zero and becomes (RG) irrelevant for all driving values. This point marks the transition to fully free growth, II $\rightarrow$ III in our language, which is also manifested by $C(r)$. This is consistent with the II $\rightarrow$ III transition being a purely thermal phenomenon and hence independent of the driving. Alternatively, we can say that the II $\rightarrow$ III transition is to an infinitely rough growing interface, which averages the periodic potential to zero. We have attempted to formalize our ideas in the framework of the overdamped limit, and we have found that if the force is introduced adiabatically, it renormalizes independently of the potential strength, much in the same way we have described. Note that below the KT transition there may be other transitions that RG is incapable of describing. Furthermore, the two candidate transitions are also related to dynamical symmetry changes in motion.

\section{CONCLUSIONS}

In summary, our large-scale LD simulations strongly indicate that the equilibrium roughening transition splits into two transitions (or crossovers) in the presence of driving. We have provided a physical interpretation of our numerical simulations that is consistent with an $\mathrm{RG}$ picture. It is important to note that the framework we provide here is also consistent with experimental results, in particular regarding the dependence of the growth rate on temperature: See, e.g., Fig. 5 in Ref. 8, which may provide direct evidence for the $\mathrm{I} \rightarrow \mathrm{II}$ crossover; oscillatory growth has also been demonstrated experimentally. ${ }^{21}$ From this viewpoint, the notion of a smeared transition ${ }^{7,10}$ is incomplete for understanding the dynamics of surface growth. Instead, a picture like the one we propose in terms of two crossovers better describes the physics.

We suggest that this transition splitting scenario may be quite general in this class of systems. Simulations of a discrete Kardar-Parisi-Zhang ${ }^{18}$ model show ${ }^{19}$ a crossover similar to II $\rightarrow$ III, identified with the onset of the rough phase, as the coefficient of the nonlinear term in that model is increased; this coefficient plays the same role as our driving $I$ on a more coarse-grained scale. ${ }^{10,19}$ Also, recent Monte Carlo simulations on a model for molecular beam epitaxy ${ }^{20}$ exhibit oscillatory growth and roughness regimes very similar to the ones we have reported. A very interesting related question is the possible connection of our results to claims of splitting of the $\mathrm{KT}$ transition into two (one $\mathrm{KT}$ and one Ising-like), in dense Coulomb gases or symmetry-broken $X Y$ magnets. ${ }^{22}$ Clearly, further theoretical studies are needed to establish rigorously the validity of our claims, for example, exploiting mappings to equivalent equilibrium models in three dimensions. ${ }^{23}$ However, it is also essential to seek experimental results directed to clarifying these points. We stress that our simulations and their interpretation lead to several experimentally accessible predictions: (i) the $\mathrm{I} \rightarrow \mathrm{II}$ crossover temperature decreases with increasing driving, whereas $\mathrm{II} \rightarrow \mathrm{III}$ is insensitive to driving; (ii) roughness is independent of driving in region I; and (iii) growth is oscillatory with period and amplitude decreasing (and growth rate increasing) with driving in region II. There is still one further prediction, namely, the I $\rightarrow$ II crossover disappears above a critical value of the driving; larger drivings would suppress region $I$. This is the case in our simulations above $I \simeq 0.6$. (see, e.g., Fig. 2 in Ref. 11 and comments therein). Experiments designed to test these ideas are possible with currently available techniques: Oscillatory growth and roughness can be observed by means of scanning tunneling microscopy, atomic force microscopy, reflection high-energy electron diffraction, or by other spectroscopic methods. ${ }^{21,24}$ We hope that this work stimulates further research along these lines.

\section{ACKNOWLEDGMENTS}

It is a pleasure to thank F. Falo, J. A. Cuesta, and P. S. Lomdahl for valuable discussions. A. S. is partially supported by DGICyT (Spain) Grant No. PB92-0248, by MEC (Spain)/Fulbright, and by the European Union Network ERBCHRXCT930413. Work at Los Alamos is performed under the auspices of the U.S. DOE.
${ }^{1}$ Solids Far From Equilibrium: Growth, Morphology and Defects, edited by C. Godrèche (Cambridge University Press, New York, 1991).

${ }^{2}$ J. D. Weeks and G. H. Gilmer, Adv. Chem. Phys. 40, 157 (1979); H. van Beijeren and I. Nolden, in Structure and Dynamics of Surfaces, edited by W. Schommers and P. Von Blackenhagen, Topics in Current Physics Vol. 43 (Springer, Berlin, 1987).

${ }^{3}$ J. M. Kosterlitz and D. J. Thouless, J. Phys. C 6, 1181 (1973); J. M. Kosterlitz, ibid. 7, 1046 (1974).

${ }^{4}$ S. T. Chui and J. D. Weeks, Phys. Rev. Lett. 40, 733 (1978).
${ }^{5}$ T. Ohta and K. Kawasaki, Prog. Theor. Phys. 60, 365 (1978).

${ }^{6}$ Y. Saito, in Ordering in Strongly Fluctuating Condensed Matter Systems, edited by T. Riste (Plenum, New York, 1980); Z. Phys. B 32, 75 (1978).

${ }^{7}$ P. Nozières and F. Gallet, J. Phys. (Paris) 48, 353 (1987).

${ }^{8}$ F. Gallet, S. Balibar, and E. Rolley, J. Phys. (Paris) 48, 369 (1987).

${ }^{9}$ T. Hwa, M. Kardar, and M. Paczuski, Phys. Rev. Lett. 66 441 (1991).

${ }^{10}$ S. Balibar and J. P. Bouchaud, Phys. Rev. Lett. 69, 862 (1992); L. Mikheev, ibid. 71, 2347 (1993); M. Rost and 
H. Spohn, ibid. 72, 784 (1994); Phys. Rev. E 49, 3709 (1994); T. Hwa, M. Kardar, and M. Paczuski, Phys. Rev. Lett. 72, 785 (1994).

${ }^{11}$ F. Falo, A. R. Bishop, P. S. Lomdahl, and B. Horovitz, Phys. Rev. B 43, 8081 (1991).

${ }^{12}$ Z. J. Wang, P. S. Lomdahl, and A. R. Bishop (unpublished).

${ }^{13}$ C. C. Hsu, Y. C. Lu, J. B. Xu, and I. H. Wilson, Appl. Phys. Lett. 64, 1959 (1994); C. C. Hsu, J. B. Xu, and I. H. Wilson, ibid. 64, 2105 (1994).

${ }^{14}$ About 2 CPU ms per lattice site per unit time on a SparcStation 2000 or a HP $9000 / 715 / 75$.

${ }^{15}$ H. S. Greenside and E. Helfand, Bell Syst. Tech. J. 60, 1927 (1981).

${ }^{16}$ N. Grønbech-Jensen, A. R. Bishop, F. Falo, and P. S. Lomdahl, Phys. Rev. B 45, 10139 (1992); ibid. 46, 11149 (1992).

${ }^{17}$ S. E. Trullinger, M. D. Miller, R. A. Guyer, A. R. Bishop,
F. Palmer, and J. A. Krumhansl, Phys. Rev. Lett. 40, 206 (1978); M. Buttiker and R. Landauer, Phys. Rev. A 23, 1397 (1981).

${ }^{18}$ M. Kardar, G. Parisi, and Y. C. Zhang, Phys. Rev. Lett. 56, 889 (1986).

${ }^{19}$ H. Guo, B. Grossmann, and M. Grant, Phys. Rev. Lett. 64, 1262 (1990); B. Grossmann, H. Guo, and M. Grant, Phys. Rev. A 43, 1727 (1991).

${ }^{20}$ S. Pal and D. P. Landau, Phys. Rev. B 49, 10597 (1994).

21 J. Villain, J. Phys. (France) I 1, 19 (1991).

22 J.-R. Lee and S. Teitel, Phys. Rev. Lett. 64, 1483 (1990).

${ }^{23}$ R. Eykholt, A. R. Bishop, P. S. Lomdahl, and E. Domany, Physica D 23, 102 (1986).

${ }^{24}$ Mechanisms of Thin Film Evolution, edited by S. M. Yalisove, C. V. Thompson, and D. J. Eaglesham, MRS Proceedings No. 317 (Materials Research Society Proceedings, Pittsburgh, 1994). 